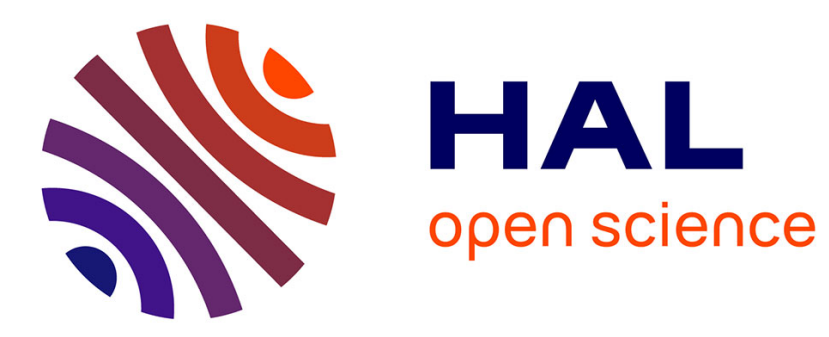

\title{
Ultrasound scattering by buoyancy driven flows
}

\author{
J. Pinton, C. Laroche, S. Fauve, Christian Baudet
}

\section{To cite this version:}

J. Pinton, C. Laroche, S. Fauve, Christian Baudet. Ultrasound scattering by buoyancy driven flows. Journal de Physique II, 1993, 3 (6), pp.767-773. 10.1051/jp2:1993166 jpa-00247870

\section{HAL Id: jpa-00247870 https://hal.science/jpa-00247870}

Submitted on 1 Jan 1993

HAL is a multi-disciplinary open access archive for the deposit and dissemination of scientific research documents, whether they are published or not. The documents may come from teaching and research institutions in France or abroad, or from public or private research centers.
L'archive ouverte pluridisciplinaire HAL, est destinée au dépôt et à la diffusion de documents scientifiques de niveau recherche, publiés ou non, émanant des établissements d'enseignement et de recherche français ou étrangers, des laboratoires publics ou privés. 
Classification

Physics Abstracts

$43.85-47.25$

\title{
Short Communication
}

\section{Ultrasound scattering by buoyancy driven flows}

\author{
J. F. Pinton, C. Laroche, S. Fauve and C. Baudet \\ Laboratoire de Physique, Ecole Normale Supérieure de Lyon, 46 Allée d'Italie, 69364 Lyon, \\ France \\ (Received 18 January 1993, accepted 5 April 1993)
}

\begin{abstract}
We show experimentally that the scattering of sound by temperature inhomogeneities may be used as a non-intrusive method to investigate the space-time characteristics of buoyancy-driven flows. We study the thermal plume generated in air by a horizontal hot wire as a test flow: we show that the onset of time-dependent instabilities as well as the advection of thermal inhomogeneities by the mean flow can be studied with our measurement technique.
\end{abstract}

\section{Introduction.}

It has been known for a long time that waves can be used to probe a medium which interacts with them. In the case of turbulent flows, scattering of sound has been studied first by Obukhov [1] and further important developments were made by Kraichnan [2] and Lund and Rojas [3]. When the flow is generated by thermal convection, one might expect that the main scattering mechanism is the one due to temperature fluctuations which lead to fluctuations in the velocity of sound [4]. A widely used model is thus a wave equation with a random propagation velocity [5]. However it has been shown recently that advection of temperature inhomogeneities by the sound wave velocity also contributes at leading order to the scattering process [6]. In the Born approximation, the scattered pressure was found to be proportional to the Fourier transform of the temperature fluctuations, which is a familiar result when scattering processes are involved. Instead of studying the statistical properties of the transmitted sound [7], we use the scattered sound-wave in order to obtain a direct measurement of the temperature fluctuations in Fourier space.

\section{Experimental set-up.}

We study the thermal plume generated in air by the convective instability above a horizontal heated wire. The wire is $0.5 \mathrm{~mm}$ in diameter and $33 \mathrm{~cm}$ in length. It is heated by a DCcurrent which dissipates a variable power $Q$ in the range $[0,3] \mathrm{W} / \mathrm{cm}$. The experimental 
set-up is sketched in figure 1 . The emitter and the receiver are both $16 \mathrm{~cm}^{2}$ Sell-type acoustic transducers [8] with a response flat within $10 \mathrm{~dB}$ in the range $[5,100] \mathrm{kHz}$. The frequency of the emitter is set by a sine-wave generator and the power spectrum of the scattered signal is monitored on a HP 3562 spectrum analyzer. The amplitude of the incident sound-wave is $P_{0} \approx 1 \mathrm{~Pa}$ which corresponds to a velocity $u_{0}^{\mathrm{s}} \approx 0.2 \mathrm{~cm} / \mathrm{s}$, thus much smaller than the typical flow velocity $(15 \mathrm{~cm} / \mathrm{s})$. The measured angular width of the diffraction lobe of the transducers is about $3^{\circ}$ at $40 \mathrm{kHz}$. In all our measurements, the frequency of the scattered sound lies within a $100 \mathrm{~Hz}$ band about the frequency of the incoming sound-wave. The flow may be synchronized at a known frequency using a vibrating ribbon, placed parallel to and slightly above the heated wire. Since temperature fluctuations generate variations of the optical index of refraction, the flow can be easily visualized by projection onto a screen. The intensity at a given point on the image is recorded with a photosensitive detector for comparison with the acoustic scattering data.

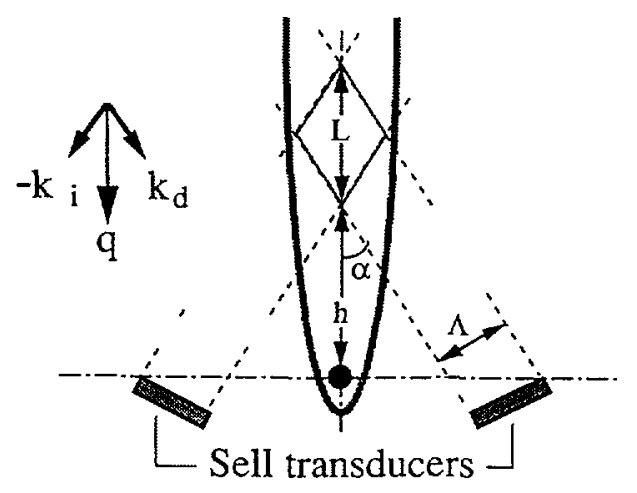

Fig. 1. - Experimental set-up. Incoming sound frequencies range between 5 and $50 \mathrm{kHz} . \alpha=15^{\circ}$, $L=60 \mathrm{~cm}, h=7 \mathrm{~cm}$. The intersection of the radiation and detection regions defines the measurement zone.

\section{Scattering by the free convective flow.}

We first describe our results on the free convective flow, i.e. in the absence of external forcing. Figure 2 shows a typical scattering spectrum (i.e. the power spectrum of the pressure-field measured by the receiver). The narrow peak at the frequency of the incoming sound is due to various reflections of the incident beam on the walls enclosing the experimental apparatus. The shifted peak exists only when the wire is heated. It is characteristic of the presence of advected temperature fluctuations since the laminar base flow is steady and thus cannot produce a frequency shift of the sound wave. These temperature fluctuations produce a phase modulation of the sound wave (the acoustic transducers are linear in pressure, i.e. phasesensitive), as well as a change in direction. Indeed, the position of this shifted peak (hereafter named the "scattering peak") is correctly given as a Doppler effect:

$$
\omega=2 \pi f=\mathbf{q} \cdot \mathbf{V}
$$


where $q=\frac{4 \pi \nu_{0}}{c_{0}} \cos \alpha$ is the scattering wavenumber, $\mathrm{V}$ is the mean velocity field, $c_{0}$ and $\nu_{0}$ are the velocity and frequency of the incident sound. Refering back to figure 1 , it is easy to see that equation (1) predicts a shift to lower frequency as observed in figure 2.

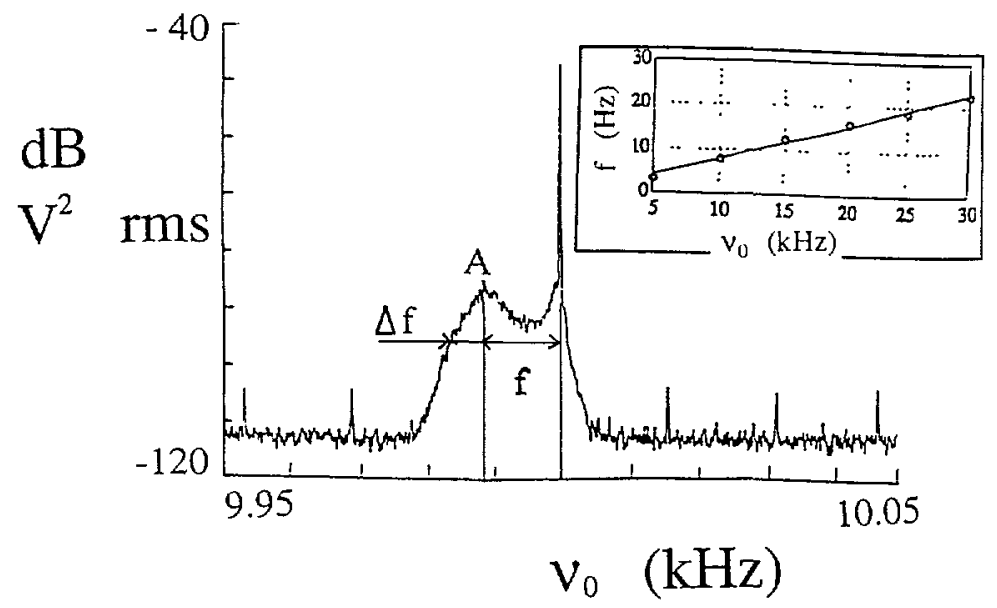

Fig. 2. - Power spectrum of scattered pressure. Heating power $Q=1 \mathrm{~W} / \mathrm{cm}$, frequency of incoming sound $\nu_{0}=10 \mathrm{kHz}$. The scattered sound is characterized by its Doppler shift $f$, the associated frequency spread $\Delta f$, and its amplitude $A$. The inset shows the linear variation of the Doppler shift with the frequency of the incoming sound.

The Doppler shift $f$ increases linearly with increasing values of the frequency $\nu_{0}$ of the incoming sound (see the inset of Fig. 2). We thus define $\bar{V}: \bar{V}=2 \pi \delta f / \delta q$. This is the mean advection velocity of the temperature fluctuations averaged over the measurement zone. When the heating power $Q$ is varied, one finds that $\bar{V}$ varies as $Q^{2 / 5}$ in good agreement with previous studies of this flow $[9,10]$.

Another quantity that can be measured on the scattered spectra is the frequency width $\Delta f$ of the scattered peak. It is found experimentally that $\Delta f$ increases linearly with the magnitude of the scattering wave-vector. Indeed, equation (1) shows that the broadening of a spectral line is: $2 \pi \Delta f=\bar{V} \Delta q+q \Delta V$, and thus has two sources. The first term comes from the lack of precision with which the scattering wave-vector is defined. This is a diffraction effect, due to the finite size of the transducers. We have $\Delta q \sim 1 / L$, independent of $q$, where $L$ is the vertical dimension of the measurement zone (see Fig. 1). The second term results from the velocity fluctuations at scale $q$ and accounts for the linear variation of $\Delta f$ with $\nu_{0}$. One can thus estimate the magnitude of the velocity fluctuations; they are found to be quite large with values of about $10 \%$ of the mean velocity.

More precisely, the scattering spectrum computed in reference [6] can be interpreted as follows [12]: for a given scattering angle, the frequency of the incoming sound selects the scattering wave vector $q$ (i.e. the scale at which the flow is probed); the amplitude of the scattered signal at the position $2 \pi|f|=q \bar{V}$ then gives the corresponding amplitude of the Fourier component $\delta \tilde{T}(q)$ of the temperature fluctuations. Figure 3 shows the spectrum of temperature fluctuations measured by varying the frequency $\nu_{0}$ of the incoming sound for various heating powers. It is found that $\delta \tilde{T}(q)$ varies as $q^{-3 / 2}$ for all $Q$. However, this result 


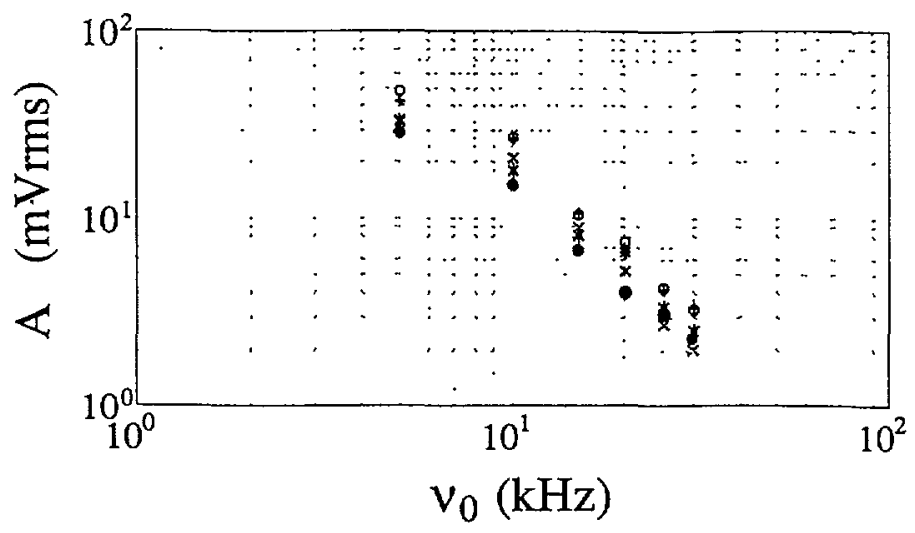

Fig. 3. - Amplitude $A$ of the scattered peak as a function of the frequency of the incoming sound. $(\bullet): Q=1 \mathrm{~W} / \mathrm{cm} ;+: Q=0.83 \mathrm{~W} / \mathrm{cm} ;(*): Q=0.66 \mathrm{~W} / \mathrm{cm} ;(\times): Q=0.55 \mathrm{~W} / \mathrm{cm} ;(0): Q=0.37$ $\mathrm{W} / \mathrm{cm}$. The slope is equal to 1.52 .

is to be considered with caution since the measurements were performed over less than one decade $\left(q \in[1.8,10.8] \mathrm{cm}^{-1}\right)$.

\section{Scattering by the externally-forced convective flow.}

When $Q$ is increased the free convective flow undergoes an instability to a time-dependent regime which displays coherent horizontal velocity oscillations at a frequency of about $3 \mathrm{~Hz}$. This frequency is detected on the scattering spectrum. We describe below the results obtained when the plume is forced by a vibrating ribbon, placed parallel to and slightly above the heated wire. A typical visualization of the forced wake is shown by the photograph of figure 4. The plume oscillatory response is large for forcing frequencies $f_{\mathrm{e}}$ between $1 \mathrm{~Hz}$ and $7 \mathrm{~Hz}$, as observed previously by Pera et al. [11]. All of our measurements are made with the same heating power $Q=0.4 \mathrm{~W} / \mathrm{cm}$, for $\alpha=30^{\circ}$.

A typical scattering spectrum is presented in figure 5. In comparison to the scattering spectrum in the absence of forcing, three effects are readily observed:

(i) the spectrum now consists of a series of discrete peaks, shifted from the incoming sound frequency by a multiple of the forcing frequency. The signal given by the photosensitive detector displays a spectrum with all the corresponding frequencies. A large number of harmonics are visible because the system is non-linear and the vibrating ribbon does not have a pure sine motion as we have verified independently.

(ii) The level of noise between the peaks is strongly decreased by the external forcing. This occurs in many open flows. The forced unstable mode grows at the expense of the others or, in other words, the vibrating ribbon synchronizes the temperature fluctuations which would otherwise happen at random.

(iii) The shifted peak corresponding to the forcing frequency is not necessarily the largest one. This is due to the spectral nature of the measurement: the scattered pressure measures the amplitude of the Fourier transform $\delta \tilde{T}$ of the temperature fluctuations around $q=\frac{4 \pi \nu_{0}}{c} \cos \alpha$, the location of which on the spectrum is given by the Doppler shift $2 \pi f=q . \mathbf{V}$. As the frequency of the incoming sound is increased, larger wave-numbers are probed so that the 


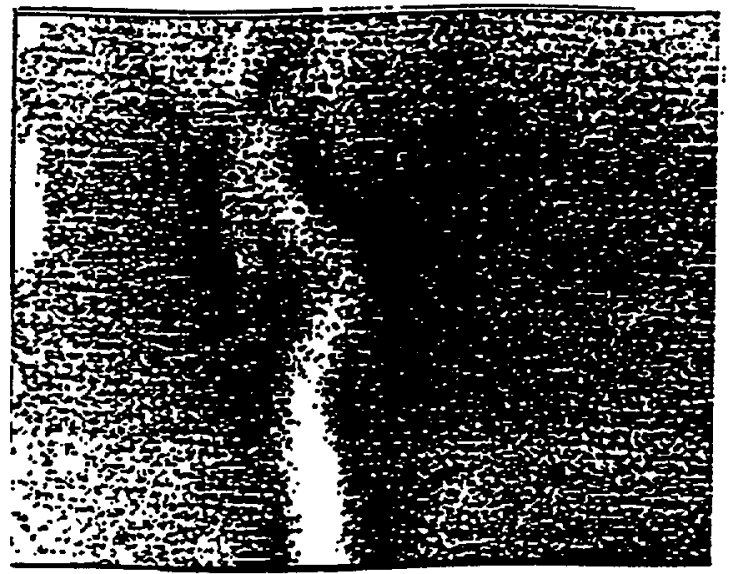

Fig. 4. - Visualization of the forced-wake. $Q=0.4 \mathrm{~W} / \mathrm{cm}, f_{e}=3 \mathrm{~Hz}$. A digital thresholding has been applied to the original picture.

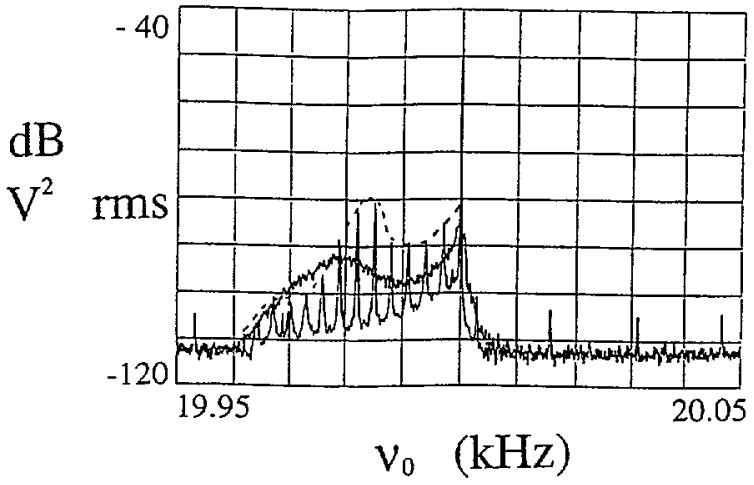

Fig. 5. - Scattering spectrum of forced wake (curve with discrete peaks). $Q=0.4 \mathrm{~W} / \mathrm{cm}, f_{\mathrm{e}}=3 \mathrm{~Hz}$, $\nu_{0}=20 \mathrm{kHz}$ - the broken curve is the envelope of the amplitude. The smooth curve is the spectrum obtained in similar conditions without forcing.

maximum amplitude is found for higher harmonics. This explains why the envelope of the visible harmonics looks like the scattering spectrum in the case of the free plume. In addition, for the same value of the frequency of the incoming sound, one observes that the maximum amplitude of the envelope of the discrete peaks occurs for a lower frequency shift than in the case of the natural wake (see Fig. 5). Since the mean scattering wavenumber is the same in both cases, this means that the mean vertical advection velocity of temperature fluctuations is lower when the wake is forced. The explanation is as follows: when the forcing is applied one observes that the amplitude of the horizontal oscillation of the plume is larger than in the natural wake ; since the ribbon feeds a negligible amount of energy into the flow, the vertical velocity must then be reduced accordingly.

As before the maximum of the envelope varies linearly as the frequency of the incoming sound 
is increased, allowing us to measure a mean advection velocity of temperature fluctuations for each forcing frequency (i.e. instability mode). We have found that this mean velocity is the smallest when the frequency of the vibrating ribbon is close to $3 \mathrm{~Hz}$. The oscillatory response of the plume is the largest for resonant forcing and draws the largest amount of energy from the base flow. Consequently, the vertical velocity has the lowest value. The $3 \mathrm{~Hz}$ value found in our experiment is in good agreement with the numerical simulations of Pera et al. [11].

\section{Discussion.}

We have shown that several features of the studied convective flow can be observed using ultrasound scattering. Let us discuss the advantages and the limitations of this experimental technique.

First, it is a spectral measurement. A scattering wave-vector $q$ is selected by choosing the scattering angle $\theta$ and the frequency $\nu_{0}$ of the incoming sound-wave. Thus, the flow can be easily probed at a chosen scale, up to a precision limited by diffraction which can be modeled and accounted for [12].

Without mean flow, the frequency spectrum of the scattered pressure has been computed using the Born approximation in reference [6]:

$$
P_{\text {scat }}\left(\mathbf{r}, \nu+\nu_{0}\right)=\frac{\rho_{0} u_{0}^{\mathbf{s}} \pi^{2} \nu^{2}}{c_{0} T_{0}} \frac{\mathrm{e}^{i \nu r / c_{0}}}{r} \cos \theta \delta \tilde{T}(\mathbf{q}, \nu)
$$

where $\rho_{0}$ and $T_{0}$ are the mean density and temperature of the fluid, and $c_{0}$ the corresponding sound velocity. The above expression shows that without a mean flow and if the temperature field is stationary, the scattered sound has the same frequency as the incoming wave. In any actual experiment the signal is then lost in the echos of the incoming sound which in our experiment are always at least $30 \mathrm{~dB}$ above the amplitude of the scattered sound.

The mean flow effect can be taken into account in (2) using the following simple argument. The incoming frequency, in the reference frame of a temperature inhomogeneity moving with constant velocity $\mathbf{V}$, is Doppler shifted by $\Delta \omega_{1}=-\mathbf{k}_{i} . \mathbf{V}$. In the same manner the scattered sound is detected with a Doppler shift $\Delta \omega_{2}=\mathbf{k}_{d} . \mathbf{V}$. The net frequency shift is $\frac{1}{2 \pi} \mathbf{q} . \mathbf{V}$, so that $P_{\text {scat }}$ in (2) should be replaced by $P_{\text {scat }}\left(\mathbf{r}, \nu+\frac{1}{2 \pi} \mathbf{q} \cdot \mathbf{V}+\nu_{0}\right)$. In the case of random temperature inhomogeneities advected in a flow $\mathbf{v}=\mathbf{V}+\mathbf{u}$, the scattered spectrum consists of a peak centered around $\nu-\nu_{0}=\frac{1}{2 \pi} \mathbf{q} . \mathbf{V}$, with a width $\frac{1}{2 \pi} \mathbf{q} \cdot \mathbf{u}$ (neglecting diffraction). Thus, the analysis of the scattered spectrum is simplified when $u \ll V$, a condition similar to the Taylor hypothesis used when deriving spatial information from local probes such as hot-wire anemometers.

When the inhomogeneities advected by a constant mean flow are triggered by a coherent oscillation at frequency $f$, they generate a spatial periodicity $V / f$. The scattered spectrum displays a peak at frequency $\nu-\nu_{0}=f$, observed only when $q=2 \pi f / V$ in the absence of diffraction. In the present experiment, the vertical velocity above the plume varies rather rapidly so that this resonance condition is not observed.

Finally, the angular dependence in equation (2) shows the existence of a backscattered wave. It is thus simple to discriminate scattering by temperature inhomogeneities from scattering due to the vorticity which presents no backscattering [13]. In this respect, we believe that ultrasound scattering could be usefully applied to the study of turbulent flows. It provides a 
local measurement in Fourier space, thus complementary to hot-wire measurements which are local in real space.

\section{Acknowledgements.}

We acknowledge very useful discussions with Fernando Lund. This work has been partly supported by the DRET with contract 90/194 and by the EEC with contract CI1-CT91-0947.

\section{References}

[1] Obukhov A. M., Dokl. Akad. Nauk. SSSR 30 (1941) 611.

[2] Kraichnan R. H., J. Ac. Soc. Am. 25 (1953) 1096-1109.

[3] Lund F. and Rojas C., Physica D 37 (1989) 508-514.

[4] Monin A. S., Soviet Phys. Ac. 7 (1962) 370-373.

[5] Neubert J. A. and Lumley J. L., J. Ac. Soc. Am. 48 (1970) 1212-1218.

[6] Contreras H. and Lund F., Phys. Lett A149 (1990) 127-130.

[7] Blanc-Benon Ph. and Juve D., AIAA 11th Aeroacoustics Conference, (1987) pp.1-7 and references therein.

[8] Anke D., Acustica 30 (1974) 30.

[9] Gebhart B., Pera L. and Schorr A. W., Int. J. Heat Mass Transfer 13 (1970) 161-171.

[10] Forstrom R. J. and Sparrow E. M., Int. J. Heat Mass Transfer 10 (1967) 321-331.

[11] Pera L. and Gebhart B., Int. J. Heat Mass Transfer 14 (1971) 975-984.

[12] Pinton J.-F., Thesis, University of Lyon I (1992).

[13] Baudet C., Ciliberto S. and Pinton J.F., Phys. Rev. Lett. 67 (1991) 193-195. 